\title{
Komparative Zeitreihenanalyse der lexikalischen Stabilität und Emotion in österreichischen Korpusdaten
}

\author{
Bettina M. J. Kern \\ University of Vienna \\ bettina2.kerneunivie.ac.at
}

Andreas Baumann

University of Vienna

andreas. baumanneunivie.ac.at

\author{
Klaus Hofmann \\ University of Vienna \\ klaus.hofmanneunivie.ac.at
}

\author{
Tanja Wissik \\ Austrian Academy of Sciences \\ Tanja.Wissik@oeaw.ac.at
}

\begin{abstract}
Anhand von zwei unterschiedlich aufgebauten Textkorpora wird untersucht, inwiefern sich lexikalischer Gebrauch und der emotionale Gehalt im Diskurs über politische Parteien in österreichischen Zeitungsbeiträgen und im Diskurs der Parteien selbst in ihren Reden vor dem österreichischen Nationalrat über die Zeit verändern. Welche Charakteristika des Diskurses verändern sich, auf welche Faktoren sind diese Dynamiken zurückzuführen und kann dies kontrastiv anhand von unterschiedlich aufgebauten Textkorpora untersucht werden? Im vorliegenden Beitrag wird anhand zweier Teilstudien diesen Fragen nachgegangen. Die beiden Teilstudien wenden eine komparative Zeitreihenanalyse an, um die Dynamiken lexikalischer Stabilität und emotionalen Gehalts im typischen Wortgebrauch dreier österreichischer Parteien herauszuarbeiten. Für komparative Untersuchungen werden üblicherweise Vergleichskorpora verwendet; in dieser Studie wird hingegen auf sehr unterschiedlich aufgebaute diachrone Korpora zurückgegriffen: Das Austrian Media Corpus (AMC) und das Korpus der Österreichischen Parlamentsprotokolle (ParlAT). Um die Textkorpora, welche sich in ihrer Textsorte und Größe deutlich unterscheiden, als Datenbasis für die komparative Analyse nutzen zu können, wird in diesem Beitrag auf die notwendige spezielle Datenaufbereitung eingegangen und gezeigt, dass wenig datenintensive (insbesondere unüberwachte) Methoden zur quantitativen Messung von diachronen Dynamiken lexikalischen Gebrauchs eingesetzt werden können. Auf diese Weise werden auch kleine Datenmengen nutzbar gemacht. Exemplarisch werden die Methoden auf den politischen Diskurs von und über österreichische Parlamentsparteien angewandt, wobei insbesondere durch Kombination zweier psychologischer Maße (Valenz und Erregung) Rückschlüsse auf den emotionalen Status des jeweiligen Diskurses gezogen werden können.
\end{abstract}

Schlüsselwörter: Wortfelder, lexikalische Stabilität, Diachronie, Zeitreihenanalyse, Emotion

\section{Einleitung}

Diese Studie widmet sich der Analyse von Dynamiken des lexikalischen Gebrauchs sowie des emotionalen Gehalts im politischen Diskurs in zwei diachronen österreichischen Korpora der vergangenen zwei Jahrzehnte. So stellt sich etwa die Frage, ob sich der Diskurs zu bestimmten Themen auf der lexikalischen Ebene mit der Zeit verändert, welche Charakteristika des Diskurses sich verändern, welche - möglicherweise extralinguistischen - Faktoren zu diesem Wandel beitragen, und wie sich schließlich diese und andere Fragen durch die Analyse von Sprachkorpora beantworten lassen.

Die korpusbasierte Erforschung diachroner Dynamiken des Lexikons ist selbstverständlich nicht neu und blickt auf eine weitreichende Geschichte zurück. Insbesondere in den letzten Jahrzehnten wurde der Analyse historischen Wandels mittels großer Datenmengen immer größere Bedeutung beigemessen. Methodologisch reicht dies von der Messung von Worthäufigkeitsentwicklungen über die 
statistische Analyse der Koevolution mehrerer Lexeme bis hin zur Studie semantischer Entwicklungen mithilfe von Methoden des maschinellen Lernens (unter anderem: Bybee 2007; Gries und Hilpert 2008; Hamilton et al. 2016a; Hilpert und Correia Saavedra 2017; Kutuzov et al. 2018; Rosenfeld und Erk 2018).

Insbesondere letztere Methoden konnten erfolgreich angewendet werden, um lexikalische Entwicklungen über lange Zeiträume zu analysieren. So entdeckten etwa Hamilton et al. (2016b) quantitative Gesetzmäßigkeiten semantisch-lexikalischen Wandels unter Zuhilfenahme eines relativ großen, zweihundert Jahre umfassenden, englischsprachigen Korpus (Corpus of Historical American English, Davies 2010). Die Forscher griffen dabei auf Methoden des unüberwachten maschinellen Lernens, unter anderem auf das Trainieren von Wort-Embeddings, also numerischer VektorRepräsentationen zur Codierung von Wortsemantik, zurück (Erk 2012). Diese Embeddings wurden schließlich benutzt, um semantische Stabilität von Lexemen zu quantifizieren.

Die vorliegende Studie befasst sich mit dem Vergleich zweier Korpora, die eine komparative Analyse politischen Diskurses (Wodak 2010) ermöglichen, in ihrer Größe und Struktur wohl aber nicht unterschiedlicher sein könnten: Das erste der beiden Korpora, das Austrian Media Corpus (AMC; Ransmayr et al. 2017), umfasst eine große Menge von heterogenen Texten aus österreichischen Printund Onlinemedien. Das zweite Korpus, das Korpus der österreichischen Parlamentsreden (ParlAT; Wissik und Pirker 2018) ist relativ klein und setzt sich aus Reden und Wortmeldungen von Parlamentsmitgliedern zusammen. Beide Korpora umspannen einen Zeitraum von nur wenigen Jahrzehnten.

In Hinblick auf die quantitative Analyse von lexikalischen Dynamiken ist dies in zweierlei Hinsicht herausfordernd: Erstens lassen sich Methoden des maschinellen Lernens nicht ohne Weiteres auf kleinere Korpora wie das ParlAT anwenden; will man, um eine bessere Vergleichbarkeit zu gewährleisten, dieselbe Methode über Korpora hinweg anwenden, so muss sich die Methodenwahl immer am kleineren Korpus orientieren. Zweitens erfordert die relativ kurze Zeitspanne der vorliegenden Korpusdaten eine besonders sensitive Methode zur Analyse diachroner Entwicklungen. Schließlich ist es umso wahrscheinlicher, kontextuellen oder semantischen Wandel zu entdecken, je größer der Beobachtungszeitraum ist.

Ziel der vorliegenden Studie ist es somit, wenig datenintensive Methoden zur quantitativen Messung von diachronen Dynamiken lexikalischen Gebrauchs zu finden, und diese zu verwenden, um Dynamiken in unterschiedlichen Korpora miteinander zu vergleichen. Diese Dynamiken sollen in weiterer Folge interpretiert und einer tiefergehenden Analyse unterzogen werden.

In dieser Studie wird das anhand zweier aufeinander aufbauender Teilstudien demonstriert: Untersuchungsgegenstand ist der Wandel des lexikalischen Gebrauchs im Diskurs von und um drei österreichische Parlamentsparteien (ÖVP, SPÖ, FPÖ) in beiden Korpora, AMC und ParlAT. In Teilstudie 1 wird zunächst die Stabilität der Wortfelder um diese Zielentitäten (d.h. Parlamentsparteien) in AMC und ParlAT untersucht und miteinander verglichen. Dies wird einerseits mit recht einfachen, aber robusten, Methoden zur Quantifizierung sprachlichen Wandels an sich und andererseits mit Methoden der Zeitreihenanalyse umgesetzt. Es wird gezeigt, dass der lexikalische Gebrauch um die Zielentitäten teilweise starken Schwankungen unterliegt und dass sich sowohl Parallelen als auch Unterschiede zwischen den beiden Korpora identifizieren lassen.

In Teilstudie 2 werden diese Dynamiken mithilfe von Methoden der Sentimentanalyse genauer untersucht. Dabei wird auf Valenz- und Erregungslexika zurückgegriffen, also Listen von Wörtern gemeinsam mit Ratings zum jeweiligen emotionalen Status. Es wird gezeigt, dass sich ein Teil des Wandels der Wortfelder wohl auf eine Veränderung in der Polarität zurückführen lässt. Weiters legen die Resultate der Analyse einen relativ starken Einfluss von Verschiebungen in der politischen Landschaft (insbesondere wechselnde Regierungsbeteiligung) auf die Wortfelder (und deren emotionale Ausprägung) der einzelnen Parteien nahe.

Selected Papers from the workshop „Digital Lexis, and Beyond”, 45th Austrian Linguistics Conference, Dec. 2019 
In den folgenden Abschnitten werden die beiden Korpora (Abschnitt 2) und danach die beiden oben erwähnten Teilstudien mitsamt der jeweiligen Methodologie beschrieben; Abschnitt 3 umfasst Teilstudie 1 und Abschnitt 4 Teilstudie 2. Die Ergebnisse beider Teilstudien und Schlussfolgerungen für die linguistische Forschung werden schließlich in Abschnitt 5 diskutiert.

\section{Daten}

Im Folgenden werden die Daten, die diesen Teilstudien zugrunde liegen, sowie die Datenaufbereitung beschrieben.

\subsection{Austrian Media Corpus}

Das Austrian Media Corpus (AMC; Ransmayr et al. 2017) ist ein diachrones Textkorpus, das am Austrian Centre for Digital Humanities und Cultural Heritage der Österreichischen Akademie der Wissenschaften erstellt wurde und fortlaufend erweitert wird. Das Textkorpus enthält Medienbeiträge und Artikel österreichischer Zeitungen und Zeitschriften, Pressemitteilungen sowie transkribierte Fernsehinterviews und Fernsehnachrichten der letzten 30 Jahre. Mit über 10 Milliarden Tokens ist das AMC eines der größten Textkorpora im deutschen Sprachraum und das größte Textkorpus für das österreichische Deutsch. In der vorliegenden Analyse wird nur ein Teil des AMC verwendet. Zeitlich wurde dieses Subkorpus auf die Jahre 1997 bis 2016 beschränkt, die mit der Dauer von sechs aufeinanderfolgenden österreichischen Regierungsperioden übereinstimmen. Was die unterschiedlichen Textsorten betrifft, wurden für diese Studie nur die Zeitungstexte verwendet. Das Subkorpus enthält 5,5 Milliarden Tokens. Die Daten sind bereits tokenisiert, lemmatisiert und nach Wortart getaggt.

\subsection{ParlAT}

Das Korpus der österreichischen Parlamentsprotokolle ParlAT enthält die offiziellen Transkripte der Reden, die vor dem österreichischen Nationalrat gehalten werden. Derzeit deckt ParlAT die 20. bis 25. Legislaturperiode von 1996 bis 2017 ab (Wissik und Pirker, 2018). Das Korpus ist tokenisiert, lemmatisiert, und mit Wortarten annotiert. Des Weiteren sind die Daten mit einem speziellen TEIMarkup gemäß den Parla-CLARIN-Richtlinien (Erjavec und Pančur, 2019) ausgezeichnet. Dabei sind z.B. alle Reden von Abgeordneten als Äußerungen $<u>$ gekennzeichnet, und jede*r Redner*in wird entsprechend identifiziert und gekennzeichnet. Zusätzliche Kommentare und Notizen der Stenograph*innen werden ebenfalls annotiert.

Das Korpus besteht aus ungefähr 75 Millionen Tokens mit rund 600.000 Wortformen und 400.000 Lemmata. Auch hier wurden für die vorliegende Studie nur die Jahre 1997 bis 2016 in die Analyse miteinbezogen, um zeitliche Kongruenz zwischen den verwendeten Korpora zu schaffen.

\subsection{Datenaufbereitung}

Die Methoden zur Datenaufbereitung stammen aus einer früheren Arbeit (Hofmann et al. 2020). Zum besseren Verständnis der vorliegenden Studie werden sie im folgenden Abschnitt nochmals im Detail beschrieben.

Ziel der Studie ist es, die Sprache, in der über die Parteien in den Medien gesprochen wird, mit der Sprache zu vergleichen, die die Parteimitglieder selbst im Parlament verwenden. Es ergibt sich jedoch eine grundsätzliche Herausforderung hinsichtlich der Vergleichbarkeit der vorliegenden Daten: Das eine Korpus, ParlAT, besteht aus Texten einzelner Sprecher*innen, deren Parteizugehörigkeit für unsere Zwecke relevant ist. Bei dem anderen Korpus, dem AMC, ist die Identität der Verfasser*innen der Texte für uns nicht relevant. Aus diesem Grund wurden die Daten vorverarbeitet, um sie für 
vergleichende Studien aufzubereiten (zu ähnlichen Fragestellungen der Datenvergleichbarkeit mittels Datenaufbereitung siehe Lehmann und Weßels 2020).

Zunächst muss definiert werden, welche sprachliche Analyseeinheit den politischen Diskurs abbilden soll. Ausgehend von der Annahme, dass politische Themen und Konzepte am deutlichsten durch Substantive (wie Arbeit, Beschäftigung, Marktwirtschaft oder Nation) repräsentiert werden, wurde in den vorliegenden Teilstudien ausschließlich diese Wortart berücksichtigt. Zudem konnten Hamilton et al. (2016a) zeigen, dass sich semantische Veränderungen an Substantiven am stärksten niederschlagen. Daher sind sie für das vorliegende Vorhaben von besonderem Interesse.

Die Lemmata aller gebräuchlichen Substantive aus den beiden Korpora wurden extrahiert und wenig informative Wörter wie Ziffern, Monate, Wochentage sowie Beamtentitel (Stadtrat, Präsident usw.) wurden mit Stoppwortlisten herausgefiltert.

Als nächstes wurden für ausgewählte politische Parteien pro Jahr (von 1997 bis 2016) Subkorpora erstellt. Die folgenden politischen Parteien wurden dabei berücksichtigt: Österreichische Volkspartei (ÖVP), die Sozialdemokratische Partei Österreichs (SPÖ) und die Freiheitliche Partei Österreichs (FPÖ/BZÖ). Letztere umfasst sowohl die ursprüngliche Freiheitliche Partei als auch deren Splittergruppe - das Bündnis Zukunft Österreichs -, die 2005 gegründet wurde und die Regierungsrolle der FPÖ übernahm. Da es auf personeller sowie thematisch-inhaltlicher Ebene eine starke Kontinuität beider Parteien gab, werden sie nicht als getrennte Entitäten geführt, sondern unter FPÖ zusammengefasst.

Die österreichischen Grünen (Die Grünen) wurden aufgrund möglicher Verwechslungen mit der gleichnamigen deutschen Partei, über die in den österreichischen Medien auch berichtet wird, und somit im AMC enthalten ist, ausgeschlossen.

Da in den beiden Korpora AMC und ParlAT unterschiedliche Annotationsstrategien zum Einsatz kamen, und sie generell deutlich unterschiedliche Textsorten enthalten, musste zunächst eine auf beide Korpora zutreffende Definition lexikalischer Kontexte entwickelt werden.

Beim ParlAT sind die lexikalischen Einheiten von Interesse, die die politischen Akteur*innen in ihren Reden verwenden. Daher bezeichnet der lexikalische Kontext in diesem Fall die Substantive, die in den Reden der Parteimitglieder vorkommen. In diesem Fall war die Zuordnung der Kontexte zur jeweiligen Partei sehr einfach, da jede Wortmeldung auf eine*n Sprecher*in zurückgeführt werden kann und die Metadaten des ParlAT unter anderem die Parteizugehörigkeit aller Sprecher*innen enthält.

Es wurden nur Reden von gewählten Vertreter*innen aufgenommen, während verfahrensorientierte Äußerungen (z. B. von den Vorsitzenden) sowie Zwischenrufe weggelassen wurden.

Im AMC hingegen kommen nicht die Vertreter*innen politischer Parteien selbst zu Wort, sondern es wird über sie berichtet. Um also vergleichbare Subkorpora für den AMC zu erhalten, wurden die Substantive im Textmaterial um die Namen der Parteien (SPÖ, ÖVP, FPÖ/BZÖ) herum extrahiert. Die Länge dieses Kontextfensters wurde auf 20 Wörter festgelegt: 10 Wörter vor und nach dem Parteinamen. Dieser Wert entspricht dem Median der Satzlänge im ParlAT (19 Wörter). Insgesamt wurden auf diese Weise 120 Subkorpora erstellt: eines für jede Partei in jedem Jahr in den beiden Korpora AMC und ParlAT.

Durch diese Datenaufbereitung und Erstellung von Subkorpora ist zu beachten, dass es sich um kleinere Datenmengen und Subkorpora handelt als die in 2.1 und 2.2 beschriebenen Ausgangsdaten.

\section{Teilstudie 1: Lexikalische Stabilität}

Die erste Teilstudie befasst sich mit der Messung lexikalischer Stabilität. Das bedeutet, dass aus den zugrundeliegenden Subkorpora für die jeweiligen Zielentitäten charakteristische Wortfelder abgeleitet werden, deren zeitliche Entwicklung in weiterer Folge gemessen wird. Diese Entwicklung wird 
schließlich mittels Zeitreihenanalyse modelliert, was den Vergleich mehrerer Zeitreihen über Korpora hinweg ermöglicht.

\subsection{Methoden}

\subsubsection{Erstellung der Wortfelder}

Basierend auf den in 2.3 beschriebenen Subkorpora (60 in AMC und 60 in ParlAT) wurden auf folgende Weise Wortfelder zu den drei Parteien erstellt: Um die - für das jeweilige Jahr - distinktiven Wörter für eine Partei zu ermitteln, wurden zunächst für jedes Wort (a) die Häufigkeit im Subkorpus der jeweiligen Partei und (b) die Gesamthäufigkeit in den verbleibenden Subkorpora im jeweiligen Jahr ermittelt. Auf diese Häufigkeitsverteilung wurde in Folge ein $\chi^{2}$-Test angewandt. Weiters wurden nur Wörter mit einer positiven $\chi^{2}$-Statistik und statistisch signifikantem Häufigkeitsunterschied (Konfidenzniveau bei 0.95) beibehalten, also solche Wörter, die statistisch häufiger gemeinsam mit der jeweiligen Partei vorkommen als mit allen anderen Parteien. Schließlich wurden alle verbliebenen Wörter nach absteigender $\chi^{2}$-Statistik gereiht, sodass das erste Wort am charakteristischsten für die Zielentität ist, und nur die 200 am meisten distinktiven beibehalten. Diese finale Auswahl an Wörtern wurde als das charakteristische Wortfeld einer Partei in einem gegebenen Jahr definiert. Das Prozedere wurde für alle drei Zielentitäten (Parteien) und alle jahresweisen Subkorpora durchgeführt.

Es ist wichtig, festzustellen, dass obige Operationalisierung von Wortfeldern unabhängig von der Struktur der jeweiligen Korpora ist und nur auf den daraus extrahieren Subkorpora beruht. Zudem zeichnet sich die Methode dadurch aus, dass sie nicht besonders datenintensiv ist: Durch die Wahl des Distinktivitätsmaßes $\left(\chi^{2}\right.$-Statistik) können für eine Entität charakteristische Wörter schon bei relativ geringen Wortfrequenzen im jeweiligen Subkorpus ermittelt werden. Beides kommt der gegenwärtigen Studie angesichts der Datenlage entgegen.

\subsection{2 Ähnlichkeiten zwischen Wortfeldern}

Um lexikalische Stabilität der Wortfelder über die Zeit zu messen, wurden im nächsten Schritt paarweise Ähnlichkeiten zwischen jeweils zwei zeitlich aufeinanderfolgenden Wortfeldern einer Zielentität berechnet. Auch hier wurde ein vergleichsweise einfaches Maß verwendet, nämlich Jaccard-Ähnlichkeit (Jaccard similarity, Jaccard 1912). Diese misst die Größe der Schnittmenge zweier Wortfelder relativ zur Vereinigung beider Wortfelder; sie ist genau 1, wenn sich die beiden Wortfelder decken, und 0, wenn es keine Überlappung der Wortfelder gibt. Somit steht eine hohe Jaccard-Ähnlichkeit für hohe lexikalische Stabilität und umgekehrt niedrige Jaccard-Ähnlichkeit für niedrige lexikalische Stabilität, also sich über die Zeit verändernde Wortfelder. Es wurde für jede Partei und für jedes Paar zeitlich aufeinanderfolgender Wortfelder auf diese Weise die lexikalische Stabilität ermittelt.

\subsubsection{Zeitreihenanalyse}

Für jede Partei und jedes Korpus (AMC, ParlAT) wurden die jeweiligen Jaccard-Ähnlichkeiten in Zeitreihen zusammengefasst, welche in Folge durch generalisierte additive Modelle (generalized additive model, GAM, Wood 2011) modelliert wurden. GAMs eignen sich hier besonders, da sich mit dieser Modellfamilie auch nichtlineare Verläufe gut modellieren lassen, indem die Zeit als sogenannte Smooth-Prädiktorvariable integriert wird. Die Komplexität des Modells wird über einen Parameter $k$ (knot, Knoten; Anzahl der additiven Funktionskomponenten im Modell) parametrisiert. In dieser Studie wurde $k$ für jedes Modell über Akaikes Informationskriterium (AIC) optimiert. Für ein fixes $k$ findet das GAM während der Kalibrierung eine optimale Balance zwischen Anpassungsgüte und Fluktuation 
(wiggliness). Zudem wurde Autokorrelation durch autoregressive Modellierung berücksichtigt (Akaike, 1969).

Auf diese Art und Weise wurden insgesamt sechs Zeitreihen modelliert. Diese Zeitreihen decken jeweils den gesamten Zeitraum von 20 Jahren ab (und somit auch alle Legislaturperioden zwischen 1996 und 2017). Für jede dieser Zeitreihen wurden zudem 95\%-Konfidenzbänder berechnet. Diese Berechnungen wurden in R mit den Paketen mgcv (Wood, 2011) und itsadug durchgeführt (van Rij et al., 2017).

\subsection{Resultate und Diskussion}

Abbildung 1 zeigt die Zeitreihenmodelle für alle Zielentitäten in den beiden Korpora. Die vertikale Achse misst die Stabilität der Wortfelder. Aus einem Vergleich der Zeitreihen lassen sich mehrere Erkenntnisse ableiten. Zunächst unterscheidet sich die Entwicklung der Stabilität in den beiden Korpora, zumindest bei den Entitäten FPÖ und SPÖ. Erstere bleibt in lexikalischer Hinsicht im ParlAT relativ instabil und weist dort konstant hohe Veränderungen auf; im AMC hingegen wird das Wortfeld der FPÖ zunehmend stabiler.

Bei der SPÖ verhält es sich umgekehrt: Bleibt die Stabilität im AMC auf relativ gleichem Niveau, so lassen sich im ParlAT signifikante Entwicklungen feststellen. Hier zeichnen sich die Jahre um 2005 sehr niedrige, die Jahre um 2010 wiederum sehr hohe Stabilität auf. Auffallend an den Zeitreihen der ÖVP, schließlich, ist der parallele Verlauf in beiden Korpora. Er zeichnet sich durch relativ geringe Stabilität bis 2005 und eine Stabilisierung der Wortfelder danach aus.

FPÖ
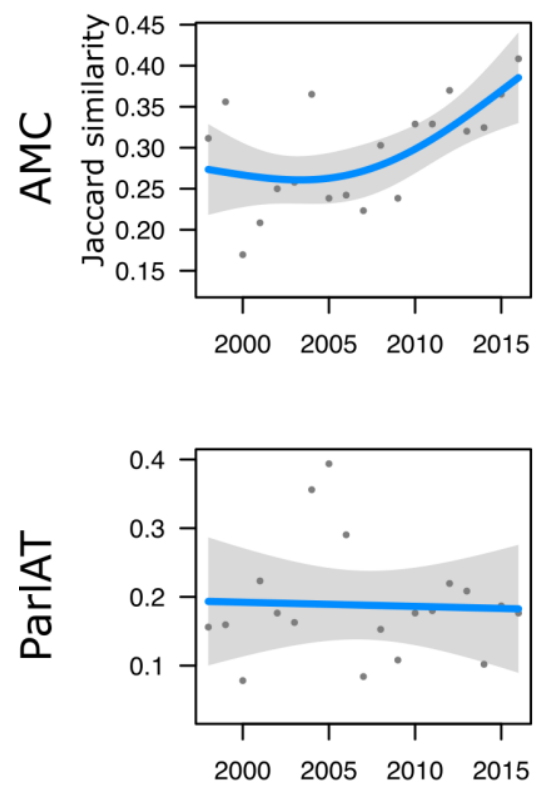

SPÖ
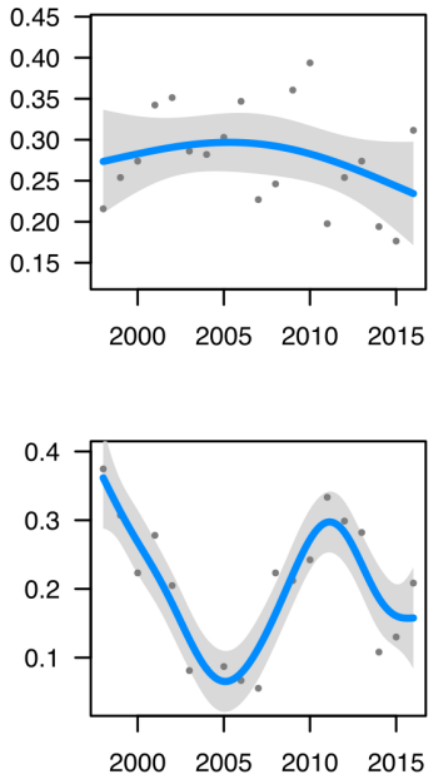

ÖVP
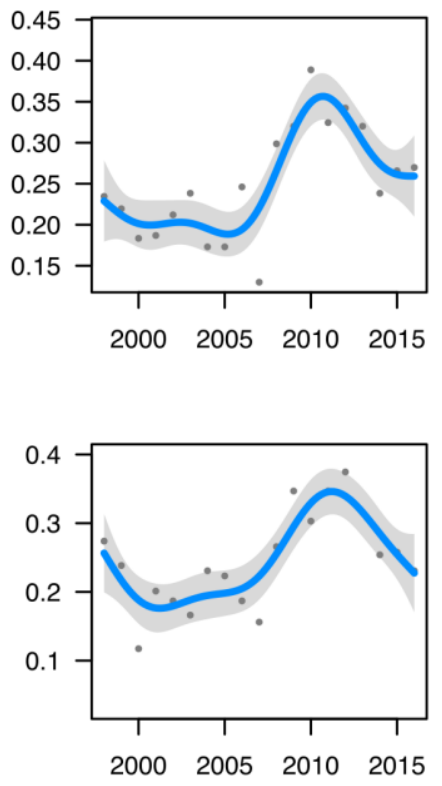

Zeit

Abbildung 1. GAM-Zeitreihen von lexikalischer Stabilität (paarweise Jaccard-Ähnlichkeiten von Wortfeldern) für alle Parteien (Spalten) in beiden Korpora (Zeilen). Niedrige Werte sprechen für stärkere Veränderung der Wortfelder. Die 95\%-Konfidenzbänder sind grau gekennzeichnet.

Im Allgemeinen lässt sich ein Abfall der lexikalischen Stabilität wohl als thematische Neuorientierung interpretieren, wohingegen ein Anstieg als thematische Festigung gedeutet werden kann. Gerade am Beispiel der SPÖ wird hier deutlich, dass eigene Themensetzung (sichtbar in ParlAT) 
und Berichterstattung (AMC) durchaus divergieren können. Eine mögliche Neuorientierung der SPÖ bis 2005 lässt sich nicht im AMC wiederfinden.

Die Frage ist, welche Aspekte des lexikalischen Gebrauchs sich nun genau verändern. In der im folgenden Abschnitt beschriebenen Teilstudie wird in dieser Hinsicht das Thema Emotion beleuchtet.

\section{Teilstudie 2: Dynamische Sentimentanalyse}

\subsection{Methoden}

Für Teilstudie 2 wurden dieselben Korpora und Methoden zur Erstellung der Wortfelder angewandt wie in Teilstudie 1 (siehe 3.1.1)

\subsubsection{Emotionaler Gehalt von Textdaten}

Die Entwicklung der Informationstechnologien führt zu immer rascher wachsenden Mengen an unstrukturierten Daten (Chen und Zhang 2014, García-Pablos und Rigau 2015), die aufbereitet und zugänglich gemacht werden müssen. Methoden zur automatisierten Verarbeitung vor allem von Textdaten sind daher ein relevantes Thema und haben im vergangenen Jahrzehnt reges Forschungsinteresse erfahren (Zhang und Liu 2014).

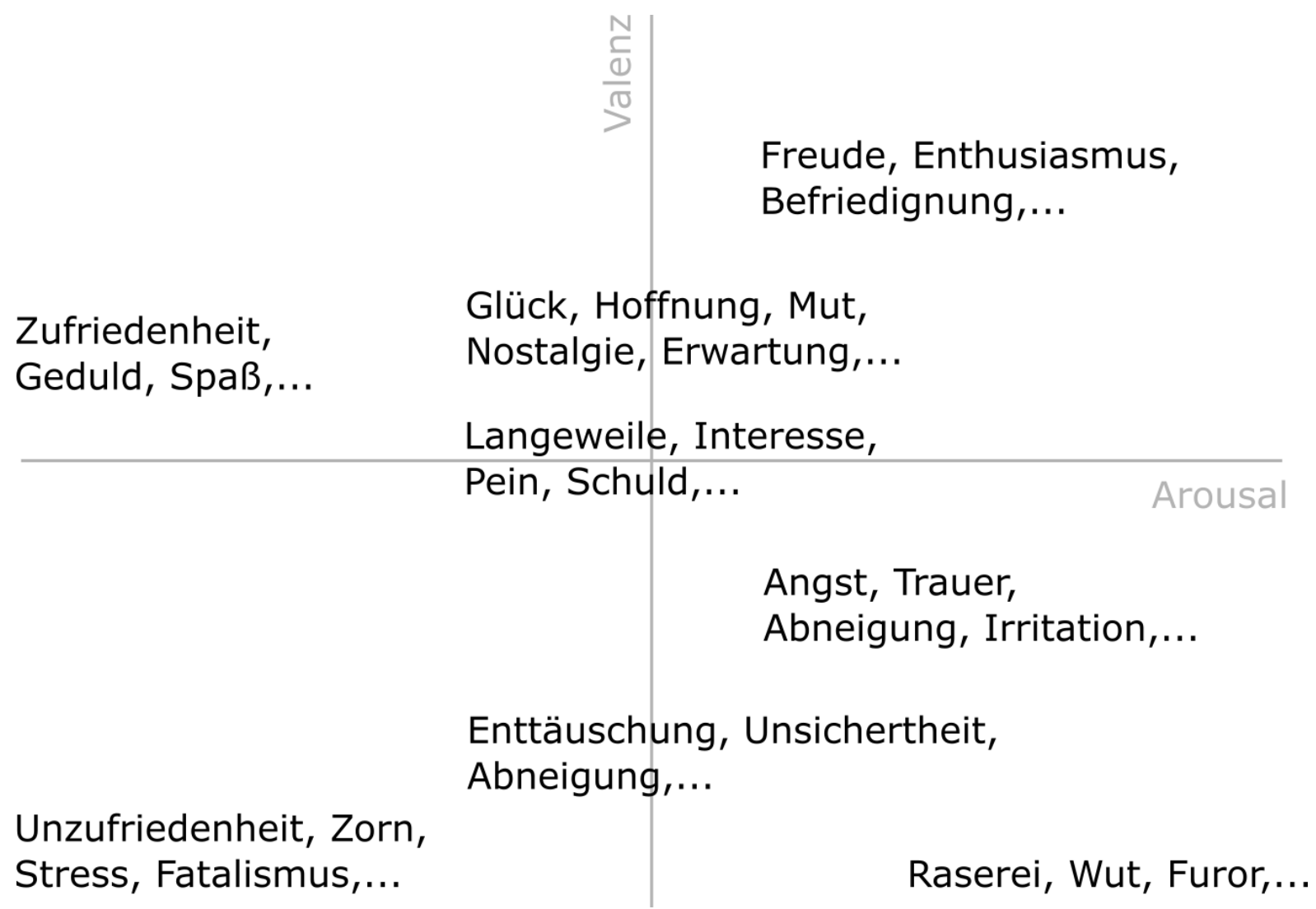

Abbildung 2. Emotionen im Circumplexmodell nach einer empirischen Clusteranalyse von Fontanari et al. (2012). Die Skizze beschreibt die ungefähren Positionen der via Umfragestudie ermittelten Emotionsgruppen im von Valenz und Arousal aufgespannten Raum.

Eine Möglichkeit zur Aufbereitung textlicher Daten ist dabei die automatisierte Erfassung des emotionalen Gehalts von Texten, die sogenannte Sentimentanalyse. Emotionen stellen für den Menschen eine wichtige Informationsquelle in der schriftlichen sowie mündlichen Kommunikation dar. 
Es gibt viele Möglichkeiten, Emotionen zu klassifizieren und zu beschreiben, entsprechend sind in der psychologischen Forschung viele Emotionstheorien bekannt. Diskrete Emotionstheorien gehen davon aus, dass Emotionen sich qualitativ voneinander unterscheiden und in distinkte Gruppen unterteilt werden können. Für die empirische Forschung und maschinelle Erkennung von Emotionen sind diese Theorien nur begrenzt nutzbar.

Dimensionale Emotionstheorien hingegen gehen davon aus, dass sich Emotionen quantitativ entlang verschiedener Dimensionen unterscheiden. Ein bekanntes Beispiel dafür ist das Circumplexmodell von Russell (1980), das menschliche Emotionen nach ihrer Ausprägung in den Dimensionen Erregung bzw. Arousal (arousal; ruhig-erregt) und Valenz (angenehm-unangenehm) verortet. Das Gefühl von Angst würde sich beispielsweise durch hohes Arousal und unangenehme Valenz auszeichnen, Unzufriedenheit ebenfalls durch unangenehme Valenz, aber ein geringes Arousal (Fontanari et al. 2012). Dies sei in Abbildung 2 schematisch dargestellt.

\subsubsection{Unüberwachte Sentimentanalyse}

Sentimentanalyse kann sowohl mit überwachten (supervised) als auch unüberwachten (unsupervised) Methoden maschinellen Lernens (machine learning) durchgeführt werden. Die vorliegende Studie beschränkt sich auf unüberwachtes Lernen. Bei dieser Methode wird der emotionale Gehalt eines Textes bestimmt, indem die Wörter mit einem Sentiment-Wörterbuch abgeglichen werden. Dieses enthält für jedes Wort Sentiment-Ratings entweder in Form von Labels (POS, NEG) oder numerische SentimentWerte (sentiment scores), die unterschiedlich skaliert sein können.

Für die vorliegende Studie wurde ein Sentiment-Wörterbuch von Köper und Schulte im Walde (2016) herangezogen. Mit 350.000 Wörtern ist es derzeit mit Abstand das umfangreichste SentimentWörterbuch für die deutsche Sprache; üblicherweise enthalten deutschsprachige SentimentWörterbücher um die 1000 bis 3000 Wörter.

Köper und Schulte im Walde (2016) haben unter Verwendung eines Algorithmus für 350.000 Wörter hinsichtlich ihrer Abstraktheit (abstractness-concreteness), Bildhaftigkeit (imageability), Erregung (arousal) und Polarität (valence) Sentiment-Werte generiert. Sie sind von 0 bis 10 skaliert, wobei hohe Werte eine höhere Erregung beziehungsweise positive Valenz widerspiegeln.

Ausgehend von der Theorie, dass Emotionen durch die Dimensionen Valenz und Erregung beschrieben werden können, wurden die Ratings für Erregung (arousal) und Polarität (valence) verwendet, um zu untersuchen, wie sich der emotionale Gehalt im Diskurs über die Parteien und der Parteien selbst über die Jahre verändert.

\subsubsection{Zeitreihenanalyse}

Basierend auf den in 3.1.1 extrahierten Wortfeldern wurde für beide Korpora (AMC und ParlAT) für jede der drei Parteien FPÖ, SPÖ und ÖVP ein durchschnittlicher Valenz- und ein durchschnittlicher Arousal-Wert für jedes Jahr von 1997 bis 2016 bestimmt. Um den Verlauf der mittleren SentimentWerte zeitlich zu modellieren, wurden generalisierte additive Modelle herangezogen, wie sie in Abschnitt 3.1.3 beschrieben sind.

Dabei wurde genau wie im Fall der Jaccard-Ähnlichkeiten Zeit als Smooth-Prädiktorvariable verwendet und ein optimaler Komplexitätsparameter $k$ über das Akaike-Informationskriterium (AIC) bestimmt. Ebenso wurden Autokorrelationen zwischen den durchschnittlichen Sentiment-Werten der aufeinanderfolgenden Jahre durch autoregressive Modellierung berücksichtigt und 95\%Konfidenzbänder berechnet.

Auf diese Weise wurden für Valenz und Arousal jeweils sechs Zeitreihenmodelle berechnet, eins für jede Partei in jedem Korpus. 


\subsection{Resultate und Diskussion}

\subsubsection{Verlauf der mittleren Valenz}

Abbildung 3 zeigt den zeitlichen Verlauf der mittleren Valenz-Werte für jede Partei in den beiden Korpora. Die vertikale Achse zeigt die durchschnittliche Valenz in den charakteristischen Wortfeldern der Zielentitäten.

Auffallend ist der Unterschied zwischen den beiden Korpora in der Streuung der Datenpunkte. Während die mittleren Valenz-Werte im ParlAT bei allen Parteien dynamische Trends aufweisen, schwankt die mittlere Valenz der im AMC verwendeten Wörter weit weniger stark, was sich in näher beieinander liegenden Datenpunkten äußert. Daraus lässt sich die Hypothese ableiten, dass in den Zeitungsartikeln mit relativ stabiler mittlerer Valenz über die Parteien berichtet wird. Im Falle der SPÖ lässt sich ein Aufwärtstrend beobachten, bei der ÖVP ein leicht fallender Trend.

Im ParlAT lassen sich hingegen wechselnde Trends beobachten. In den Reden vor dem Nationalrat schwanken die Parteien in der mittleren Valenz ihres Sprachgebrauchs.

Im Falle der FPÖ zeigt die Zeitreihenkurve ein Ansteigen der mittleren Valenz Ende der 1990er. $\mathrm{Zu}$ dieser Zeit, der 21. Legislaturperiode, gelangt die FPÖ in die Regierung. Im Jahr 2002 kommt es zur Spaltung der Partei in FPÖ und BZÖ, wobei letztere die Regierungsrolle der FPÖ übernimmt und die FPÖ selbst in der Opposition landet. An diesem Zeitpunkt sinkt die mittlere Valenz des Sprachgebrauchs der FPÖ vor dem Nationalrat stetig ab. Gegen Ende der 24. Legislaturperiode hin in den frühen 2010ern erreicht sie ihren Tiefpunkt.
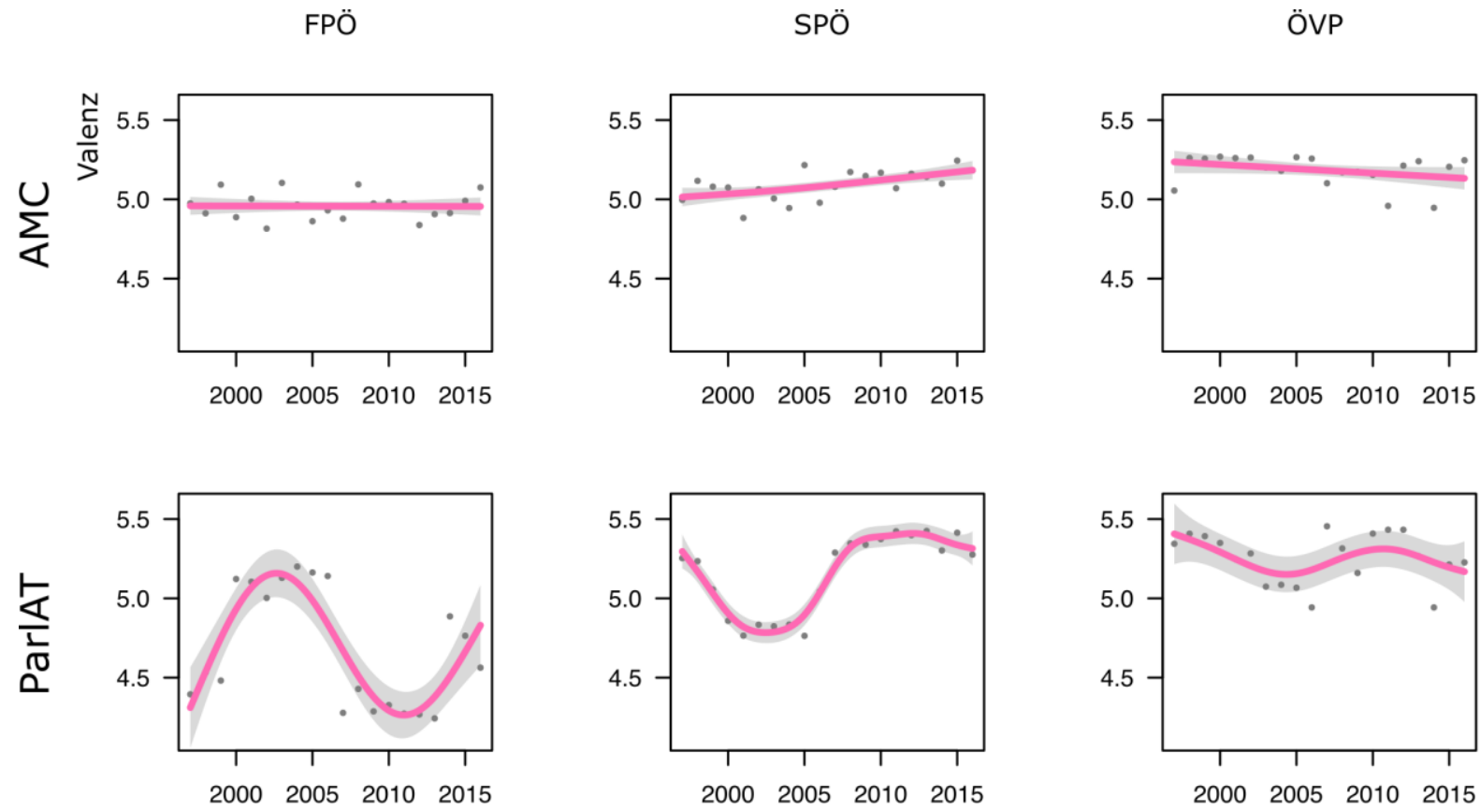

Zeit

Abbildung 3. GAM-Zeitreihen der mittleren Valenz des Sprachgebrauchs in den beiden Korpora (AMC und ParlAT) für FPÖ, SPÖ und ÖVP von 1997 bis 2016. Hohe Werte entsprechen einer positiven Valenz. Die 95\%-Konfidenzbänder sind grau gekennzeichnet.

Für die SPÖ zeichnet sich ein fast gegenläufiger Trend ab, dessen zeitlichen Verlauf man ebenfalls mit ihren politischen Rollen in Verbindung bringen kann. Zu Beginn der Zeitreihe sind die mittleren Valenz-Werte der SPÖ relativ hoch. Zu dieser Zeit, von 1996 bis 1999, der 20. Legislaturperiode, befindet sich die SPÖ gemeinsam mit der ÖVP in der Regierung. Danach, um die 
Jahrtausendwende, kommt es zu einem Absinken der mittleren Valenz-Werte der Wörter in den Reden der SPÖ. Zu dieser Zeit, der 21. und 22. Legislaturperiode von 1999 bis 2006, befindet sich die SPÖ in der Rolle der Opposition. Nach dem Ende der Koalition von ÖVP und BZÖ 2006 tritt die SPÖ wieder in die Regierung ein. Die mittleren Valenz-Werte beginnen zu dieser Zeit zu steigen und sind über die Regierungszeit der SPÖ in der 23., 24. und 25. Legislaturperiode bis zum Jahr 2017 relativ hoch.

Die ÖVP ist in allen Legislaturperioden, die unser zeitliches Subkorpus des AMC umfasst, durchgehend an der Regierung beteiligt gewesen. Analog dazu zeigen sich in der Zeitreihenanalyse der mittleren Valenz konstant hohe Werte, die wesentlich weniger stark fluktuieren als die der anderen beiden Parteien, die zwischen Oppositions- und Regierungsrolle gewechselt haben.

\subsubsection{Verlauf des mittleren Arousals}

Der zeitliche Verlauf des mittleren Arousals in den beiden Korpora über die Spanne von 20 Jahren wird in Abbildung 4 gezeigt. Insgesamt spielen sich die Schwankungen im mittleren Arousal in beiden Korpora und bei allen Zielparteien in einem viel kleineren Bereich ab, als dies bei der Valenz der Fall war.

Ähnlich wie bei der mittleren Valenz schwanken die Arousal-Werte im AMC nur innerhalb eines kleinen Bereiches und die Modellierung mittels GAMs lässt lediglich globale Trends erkennen.
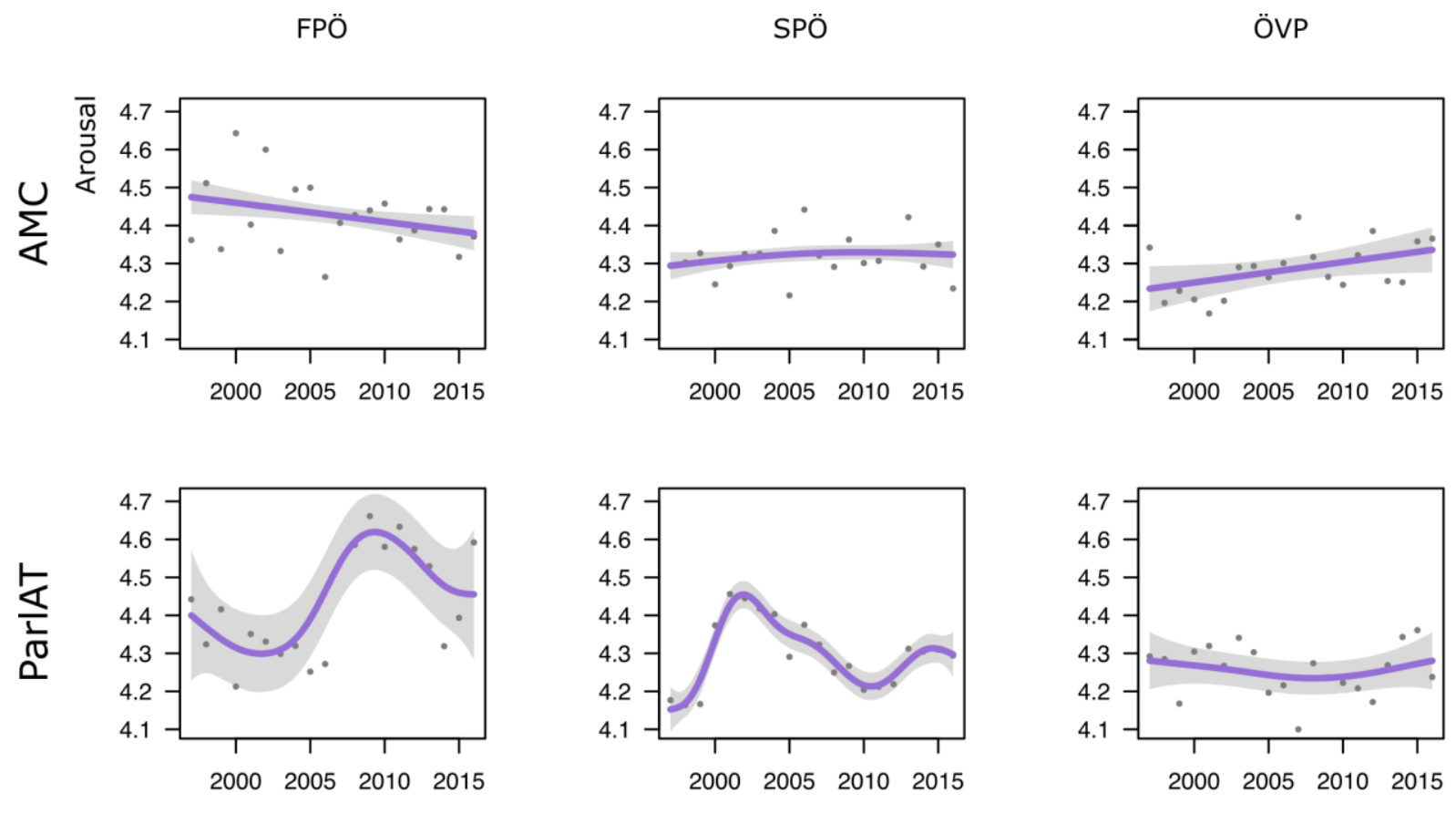

Abbildung 4. GAM-Zeitreihen des mittleren Arousals des Sprachgebrauchs in den beiden Korpora (AMC und ParlAT) für FPÖ, SPÖ und ÖVP von 1997 bis 2016. Hohe Werte entsprechen einem hohen Erregungsniveau.

Das mittlere Arousal der FPÖ im AMC liegt dabei etwas höher als bei den übrigen Zielentitäten, vor allem zu Beginn der Zeitreihe am Ende der 1990er Jahre. Zudem fluktuiert das mittlere Arousal bis Mitte der 2000er Jahre relativ stark und pendelt sich ab dann auf einem etwas niedrigeren Erregungsniveau ein. Mit Veränderungen in der politischen Rolle der FPÖ lässt sich dieser generelle Trend nicht vereinen. Ein möglicher Erklärungsansatz besteht in der zunehmenden Etablierung der FPÖ als "bürgerliche" Partei, die seit etwa 1986 in ihren Wählerstimmen in der breiten Bevölkerung zugelegt hat. 
Für die SPÖ und ÖVP ist die Schwankungsbreite des mittleren Arousals im ZeitschriftenSubkorpus des AMC sehr ähnlich. Für die ÖVP lassen die GAMs einen steigenden Trend erkennen, also eine tendenzielle Zunahme im Erregungsniveau in der Zeitungsberichterstattung über die ÖVP.

Genau wie bei den Zeitreihenmodellierung der Valenz weisen die mittleren Arousal-Werte des ParlAT stärkere Schwankungen auf als die des AMC.

Bei der FPÖ zeigt sich der tiefste Punkt des mittleren Arousals in den Reden vor dem Nationalrat in den frühen 2000er Jahren. Parallel dazu, von 1999 bis 2002, befindet sich die FPÖ in der Regierungsrolle gemeinsam mit der ÖVP. Für den darauffolgenden Zeitraum ab 2002 in der Opposition lässt die Zeitreihenanalyse ein stetiges Ansteigen der mittleren Arousal-Werte erkennen, also eine Zunahme im Erregungsniveau im Vokabular in den Reden vor dem Nationalrat.

In den 2010er Jahren kommt es dann zu einem Absinken des mittleren Arousals. Die FPÖ befindet sich zu dieser Zeit nach wie vor in der Opposition. Ein Wechsel der politischen Rolle der Zielentität kann hier nicht als Erklärungsansatz dienen.

Ähnlich wie bei der mittleren Valenz weist die Zeitreihe der SPÖ auch beim mittleren Arousal einen gegenläufigen Verlauf zu dem der FPÖ auf. Zu Beginn der Zeitreihe liegen die mittleren ArousalWerte in den Reden vor dem Nationalrat sehr tief und unterschreiten die der anderen Zielentitäten im gesamten untersuchten Zeitraum. Zu dieser Zeit, der 20. Legislaturperiode von 1996 bis 1999, hat die SPÖ eine Regierungsrolle gemeinsam mit der ÖVP inne. Parallel zum Eintritt in die Opposition Ende 1999 beginnt das mittlere Arousal im Sprachgebrauch ihrer Repräsentant*innen anzusteigen und erreicht etwa in Jahr 2002 einen Höhepunkt. Zu dieser Zeit kommt es zur Spaltung der mit der ÖVP regierenden FPÖ in BZÖ und FPÖ, wobei erstere die Regierungsfunktion der FPÖ übernimmt. Möglicherweise haben die Tumulte rund um diese Abspaltung den Oppositionsparteien mehr Angriffsfläche auf die regierenden Parteien geboten.

In den darauffolgenden Jahren sinkt das mittlere Arousal der SPÖ ab. Parallel dazu, ab dem Jahr 2006, befindet sie sich in stetiger Koalition der ÖVP. Dennoch lässt die Zeitreihe einen Wiederanstieg der mittleren Arousal-Werte ab den 2010er Jahren erkennen. Mit einem Wechsel der politischen Rolle der SPÖ lässt sich dieser Trendwechsel nicht erklären.

Bei der ÖVP liegt das mittlere Arousal über den gesamten untersuchten Zeitraum auf einem relativ zu den anderen Zielentitäten niedrigen Niveau. Es gibt zwar Schwankungen in den individuellen Datenpunkten, doch lässt sich kein starker Trend erkennen. Dies passt zu der konstanten politischen Rolle der ÖVP als Regierungspartei in diesem Zeitraum.

\subsubsection{Valenz und Arousal}

Für gewöhnlich beschränkt sich Sentimentanalyse auf die Bestimmung der Valenz des untersuchten Textes. Das Sentiment-Wörterbuch von Köper und Schulte im Walde (2016) erfasst vier Dimensionen und ist damit relativ einzigartig: Die meisten verfügbaren Sentiment-Wörterbücher decken auch nur eine Dimension ab, in den meisten Fällen Valenz. Dabei wäre es eine interessante Fragestellung, ob es einen Erkenntnisgewinn bringt, verschiedene Dimensionen von Emotion nicht isoliert zu betrachten, sondern zu vereinen. Das Circumplex-Modell von Russell (1980) geht davon aus, dass Emotion als eine Kombination aus Valenz und Arousal definiert werden kann. In der vorliegenden Studie wurden Valenzund Arousal-Verläufe modelliert und in den vorherigen Abschnitten 4.2.1 und 4.2.2 isoliert beschrieben. Es lassen sich im ParlAT jedoch auch Muster für eine kombinierte Betrachtung der mittleren Valenzund Arousal-Verläufe erkennen.

Im Fall der FPÖ lässt sich in den frühen 2000er Jahren ein Absinken des mittleren Arousals verzeichnen, zum tiefsten Punkt innerhalb der untersuchten Zeitspanne. Zeitgleich erreicht die mittlere Valenz im Sprachgebrauch vor dem Nationalrat ihren Höhepunkt. In Anlehnung an das CircumplexModell von Russell (1980) und der darauf basierenden Studie von Fontanari et al. (2012) würde man 
daraus eine ruhige, positive Emotion wie beispielsweise Zufriedenheit im Sprachgebrauch der Partei herauslesen. Zu dieser Zeit fand sich die FPÖ in der Regierung.

Dasselbe Muster zeigt sich auch bei der ÖVP. Wie in Abschnitt 4.2.1 und 4.2.2 erläutert wurde, ist die mittlere Valenz der ÖVP im ParlAT, verglichen mit den anderen beiden Parteien, konstant hoch. Gleichzeitig befindet sich das mittlere Arousal in einem konstant niedrigen Bereich. Genau wie bei der FPÖ könnte man diese Kombination der Ausprägungen im mittleren Arousal und der mittleren Valenz als ein Gefühl der Zufriedenheit im Vokabular der ÖVP-Repräsentant*innen in ihren Reden vor dem Nationalrat interpretieren. Genau wie die FPÖ befand sich die ÖVP in der Regierungsposition.

Bei der SPÖ zeigt sich für den beschriebenen Zeitraum ein gegenteiliger Verlauf, der ebenfalls zu ihren politischen Rollen in dieser Zeit passt. Im Jahr 2002 treten ÖVP und FPÖ in eine gemeinsame Koalition ein, die SPÖ wird aus der Regierungsrolle verdrängt und landet in der Opposition. Zu dieser Zeit sinkt die mittlere Valenz in den Reden vor dem Nationalrat auf den tiefsten Punkt innerhalb des untersuchten Zeitraums. Gleichzeitig steigt das Arousal auf einen Höchstpunkt. Angelehnt an Russells Circumplex-Modell und die schematische Darstellung von Fontanari et al. (2012) in Abbildung 2 würde man den Repräsentant*innen der SPÖ hier im Mittel Irritation und Wut konstatieren.

Die Kombination der Valenz- und Arousal-Dimensionen scheint also durchaus geeignet, einen explikativen Mehrwert zu liefern. Auch wenn er in diesem Fall post-hoc und rein beschreibendexplorativ ist, kann er jedoch spannende Denkanstöße und Impulse für zukünftige Hypothesengenerierung bieten.

\section{Diskussion und Schlussfolgerung}

In diesem Beitrag wurden Methoden beschrieben, die es ermöglichen, lexikalische Stabilität und emotionale Zustände von Entitäten in Texten über die Zeit zu ermitteln und diese Entwicklungen über Korpora hinweg zu vergleichen. Die Methoden wurden auf österreichische Mediendaten (AMC) sowie auf Parlamentsreden (ParlAT) angewandt.

Die Beschaffenheit dieser beiden Korpora (insbesondere ParlAT) verlangt nach relativ einfachen und wenig datenintensiven Methoden, weshalb in dieser Studie vorwiegend auf unüberwachte Ansätze zurückgegriffen wurde: Lexikalische Stabilität wurde über den Vergleich extrahierter charakteristischer Wortfelder um Entitäten operationalisiert und Werte für Arousal bzw. Valenz wurden über jeweilige Wortlisten aus den Wortfeldern errechnet. Die Entwicklungen der jeweiligen Maße wurden über Zeitreihen modelliert, was einerseits diachrone Vergleiche, andererseits aber auch Vergleiche über verschiedene Entitäten bzw. Korpora hinweg ermöglicht.

Interessant ist dabei insbesondere die Kombination der Informationen aus den Zeitreihen für Erregung und Valenz: Gemäß des in der Psychologie viel diskutierten und erweiterten CircumplexModells von Russell (1980) lassen sich Emotionen als Kombination dieser beiden Maße darstellen. Somit ermöglicht die hier vorgestellte Methode die Beobachtung des emotionalen Verlaufs von Entitäten. Dies wiederum lässt Rückschlüsse auf die Abgrenzung zeitlicher Perioden zu, in denen sich eine Entität aus lexikalisch-emotionaler Sicht konstant verhält.

Auf der inhaltlichen Ebene lässt sich aus der Anwendung der oben beschriebenen Methoden auf die österreichischen Parlamentsparteien ÖVP, SPÖ und FPÖ/BZÖ als Entitäten ableiten, dass lexikalischer und emotionaler Wandel im Sprachgebrauch der bzw. in der Berichterstattung über diese Entitäten oft mit Regierungswechseln einhergeht. Die aus Parlamentsreden abgeleiteten Wortfelder von Regierungsparteien zeichnen sich durch positive Valenz und relativ geringe Erregung aus, wohingegen die Wortfelder von Oppositionsparteien typischerweise von negativer Valenz und hoher Erregung geprägt sind. Umgekehrt erlaubt somit die Beobachtung der diachronen Entwicklung von Wortfeldern auch die Identifikation von Regierungsperioden. 
Im Gegensatz zu den Parlamentsreden weisen die Mediendaten weit weniger emotionalen Wandel auf (Abbildung 3-4). Die Tatsache, dass die Stabilität der aus dem AMC abgeleitete Wortfelder (insbesondere für FPÖ und ÖVP; Abbildung 1) sich über die letzten zwei Jahrzehnte verändert hat, deutet somit auf eine thematische Neuorientierung hin, die unabhängig von emotionalen Änderungen in der Berichterstattung zu sehen ist.

Diese inhaltlichen Erkenntnisse dienen in der vorliegenden Studie als Proof-of-Concept der vorgestellten Methoden (und sind für sich genommen natürlich nicht unerwartet und scheinen plausibel). Den Autoren scheint die Kombination dieser Methoden, insbesondere was die Erweiterung der Sentimentanalyse durch eine zusätzliche Dimension betrifft, ein erfolgversprechender Ansatz zu sein, der interessante Rückschlüsse auf den Wandel von emotionalen Aspekten des Sprachgebrauchs zulässt.

\section{Danksagung und Projektförderung}

Dieser Beitrag ist teilweise im Rahmen des DYLEN (Diachronic Dynamics of Lexical Networks) Projekts, gefördert im ÖAW go!digital Next Generation grant (GDNG 2018-020) sowie teilweise im Rahmen des DYSEN (Dynamische Sentimentanalyse als emotionaler Kompass für die digitale Medienlandschaft), gefördert durch die Stadt Wien im "Digital Humanism" Call, entstanden.

\section{Literatur}

Akaike, Hirotugu. 1969. Fitting autoregressive models for prediction. Annals of the Institute of Statistical Mathematics, 21(1). 243-247.

Bybee, Joan. 2007. Diachronic linguistics. In Geeraerts, Dirk and Hubert Cuyckens (Eds.), The Oxford Handbook of Cognitive Linguistics. 945-987. Oxford: Oxford University Press.

Chen, Philip. C. L \& Chun-Yang Zhang. 2014. Data-intensive applications, challenges, techniques and technologies: A survey on big data. Information Sciences, 275, pages 314-347.

Davies, Mark. 2010. The corpus of historical American English: COHA. Brigham: BYE, Brigham Young University.

Erjavec, Tomaž \& Andrej Pančur. 2019. Parla-CLARIN: TEI guidelines for corpora of parliamentary proceedings. In Book of abstracts of the TEI2019: What is text, really? TEI and beyond. University of Graz.

Erk, Katrin. 2012. Vector space models of word meaning and phrase meaning: A survey. Language and Linguistics Compass, 6(10). 635-653.

Fontanari, José, Bonniot-Cabanac, Marie-Claude, Michel Cabanac \& Leonid Perlovsky. 2012. A structural model of emotions of cognitive dissonances. Neural Networks, 32, 57-64.

García-Pablos, Aitor \& German Rigau. 2015. V3: Unsupervised aspect based sentiment analysis for SemEval2015 Task 12. In Proceedings of the 9th International Workshop on Semantic Evaluation (SemEval 2015). 714-718.

Gries, Stefan T. \& Martin Hilpert. 2008. The identification of stages in diachronic data: Variability-based neighbour clustering. Corpora, 3(1). 59-81. https://doi.org/10.3366/E1749503208000075

Hamilton, William L., Jure Leskovec \& Dan Jurafsky. 2016a. Cultural shift or linguistic drift? Comparing two computational measures of semantic change. In Proceedings of the Conference on Empirical Methods in Natural Language Processing. Conference on Empirical Methods in Natural Language Processing. 2116-2121.

Hamilton, William L., Jure Leskovec \& Dan Jurafsky. 2016b. Diachronic word embeddings reveal statistical laws of semantic change. In Proceedings of the 54th Annual Meeting of the Association for Computational Linguistics. Association for Computational Linguistics, arXiv preprint, arXiv:1605.09096.

Hilpert, Martin \& David Correia Saavedra. 2017. Using token-based semantic vector spaces for corpus-linguistic analyses: From practical applications to tests of theoretical claims. Corpus Linguistics and Linguistic Theory. 1-32.

Hofmann, Klaus, Anna Marakasova, Andreas Baumann, Julia Neidhardt \& Tanja Wissik. 2020. Comparing lexical usage in political discourse across diachronic corpora. In Proceedings of the Second ParlaCLARIN Workshop. 58-65.

Jaccard, Paul. 1912. The distribution of the flora in the alpine zone. New Phytologist, 11. 37-50. 
Köper, Maximilian \& Sabine Schulte im Walde. 2016. Automatically generated affective norms of abstractness, arousal, imageability and valence for 350000 German Lemmas. In Proceedings of the 10th International Conference on Language Resources and Evaluation. 2595-2598.

Kutuzov, Andrey, Lilja Øvrelid, Terrence Szymanski \& Erik Velldal. 2018. Diachronic word embeddings and semantic shifts: A survey. arXiv preprint, arXiv:1806.03537

Lehmann, Pola \& Bernhard Weßels. 2020. Different arenas, different texts, one message? What we can learn from a combined analysis of manifestos and parliamentary debates. Keynote given at the ParlaCLARIN II: LREC2020 workshop on creating, using and linking parliamentary corpora with other types of political discourse, May 11, 2020.

Ransmayr, Jutta, Karlheinz Mörth \& Matej Ďurčo. 2017. AMC (Austrian Media Corpus) - Korpusbasierte Forschungen zum österreichischen Deutsch. In Claudia Resch and Wolfgang U. Dressler (eds.), Digitale Methoden der Korpusforschung in Österreich (Veröffentlichungen zur Linguistik und Kommunikationsforschung Nr. 30). 27-38. Wien: Verlag der Österreichischen Akademie der Wissenschaften.

Rij, Jacolien van, Martijn Wieling, Harald R. Baayen \& Hedderik van Rijn. 2017. itsadug: Interpreting Time Series and Autocorrelated Data Using GAMMs. R package version 2.3.

Rosenfeld, Alex \& Katrin Erk. 2018. Deep neural models of semantic shift. In Proceedings of the 2018 Conference of the North American Chapter of the Association for Computational Linguistics: Human Language Technologies. 474-484.

Russell, James A. 1980. A circumplex model of affect. Journal of Personality and Social Psychology, 39. 11611178.

Wissik, Tanja \& Hannes Pirker. 2018. ParlAT beta Corpus of Austrian Parliamentary Records. In Proceedings of the LREC 2018 Workshop 'ParlaCLARIN: LREC2018 workshop on creating and using parliamentary corpora'. 20-23.

Wodak, Ruth. 2010. The discourse of politics in action: Politics as usual. Basingstoke: Palgrave Macmillan.

Wood, Simon N. 2017. Generalized Additive Models: An Introduction with R. London: Chapman \&Hall / CRC.

Zhang, Lei \& Bing Liu. 2014. Aspect and entity extraction for opinion mining data mining and knowledge discovery for big data. In Wesley W. Chu (Ed.) Data Mining and Knowledge Discovery for Big Data. Studies in Big Data, 1. 1-40. Springer, Heidelberg.

\section{Anhang}

Daten und Code für die vorliegende Studie befinden sich in folgendem GitLab-Repositorium: https://gitlab.com/bettina mj_kern/lexical-stability-and-emotion-in-two-diachronic-corpora

Weiters finden sich hier die Tabellen mit den bereinigten $R^{2}$-Werten:

\begin{tabular}{lllr}
\hline & & bereinigtes $^{2}$ \\
\hline Jaccard-Ähnlichkeit & AMC & FPÖ & 0.367 \\
& & SPÖ & 0.069 \\
& ÖVP & 0.678 \\
\cline { 2 - 4 } & & FPÖ & -0.056 \\
& & SPÖ & 0.756 \\
& ÖVP & 0.698
\end{tabular}

Tabelle 1. Bereinigte $R^{2}$-Werte für die GAM-Modelle der Jaccard-Ähnlichkeiten aufeinanderfolgender Jahre für die drei Parteien in AMC und ParlAT 


\begin{tabular}{lllr}
\hline & & & bereinigtes $R^{2}$ \\
\hline Valenz & AMC & FPÖ & -0.056 \\
& SPÖ & 0.297 \\
& ÖVP & 0.032 \\
\cline { 2 - 4 } & ParlAT & FPÖ & 0.692 \\
& SPÖ & 0.927 \\
Arousal & ÖVP & 0.256 \\
& & FPÖ & 0.028 \\
& & SPÖ & -0.027 \\
& & ÖVP & 0.190 \\
\cline { 2 - 4 } & ParlAT & FPÖ & 0.441 \\
& SPÖ & 0.833 \\
& ÖVP & 0.063 \\
\hline
\end{tabular}

Tabelle 2. Bereinigte $R^{2}$-Werte für die GAM-Modelle von Valenz und Arousal für die drei Parteien in AMC und ParlAT. 